\title{
REIC/Dkk-3 stable transfection reduces the malignant phenotype of mouse prostate cancer RM9 cells
}

\author{
JIE CHEN $^{2}$, MASAMI WATANABE ${ }^{1,2}$, PENG HUANG $^{2}$, MASAKIYO SAKAGUCHI $^{4}$, \\ KAZUHIKO OCHIAI ${ }^{1}$, YASUTOMO NASU ${ }^{2}$, MAMORU OUCHIDA ${ }^{3}$, NAM-HO HUH ${ }^{4}$, \\ KENJI SHIMIZU $^{3}$, YUJI KASHIWAKURA ${ }^{1}$, HARUKI KAKU ${ }^{1,2}$ and HIROMI KUMON ${ }^{1,2}$ \\ ${ }^{1}$ Innovation Center Okayama for Nanobio-Targeted Therapy; Departments of ${ }^{2}$ Urology; \\ ${ }^{3}$ Molecular Genetics; ${ }^{4}$ Cell Biology, Graduate School of Medicine, Dentistry and \\ Pharmaceutical Sciences, Okayama University, Okayama, Japan
}

Received May 26, 2009; Accepted July 23, 2009

DOI: $10.3892 /$ ijmm_00000293

\begin{abstract}
The reduced expression in immortalized cells (REIC)/Dickkopf (Dkk)-3, a member of the Dkk gene family, is a tumor suppressor in a broad range of cancers. REIC/Dkk-3 transfected stable clones of mouse prostate cancer RM9 cells (RM9-REIC) and the empty vector-transfected control clone cells (RM9-EV) were established. Clones were used to evaluate the anti-cancer effects and a proteomics analysis of REIC/Dkk-3 continuous expression was performed. The RM9-REIC cells show a feeble appearance and the cell membrane shows irregular buds known as blebs. In vitro cell proliferation was significantly suppressed in RM9-REIC clones in comparison to the control. The apoptosis assay was done under standard culture conditions and RM9-REIC showed a higher incidence of apoptosis. The RM9-EV and RM9-REIC cells were orthotopically implanted into a C57BL/6 mouse prostate. After 2 weeks, the tumor growth was significantly inhibited in RM9-REIC cells in comparison to the control. Two-dimensional gel electrophoresis was used to examine the modification of protein expression by the gene transfection. The analysis with mass spectrometry disclosed that expression of peroxiredoxin-1, GST-P1, transgelin-2, MRP-L12, ARD, GRP78 and Sorcin were increased and eEF1A-1 and cyclophilin-40 protein were decreased in RM9-REIC cells. Therefore, REIC/Dkk-3 stable transfectants show a reduction of malignancy in mouse prostate cancer RM9 cells in vitro and in vivo. The result of the proteomics analysis might provide important clues to clarify
\end{abstract}

Correspondence to: Dr Masami Watanabe, Okayama University, 2-5-1 Shikata-cho, Okayama, 700-8558, Japan

E-mail: mwcorrespondence@gmail.com

Key words: reduced expression in immortalized cells, Dickkopf-3, prostate cancer, malignant phenotype, two-dimensional gel electrophoresis the anti-cancer molecular mechanism of REIC/Dkk-3 gene transfer.

\section{Introduction}

The reduced expression in immortalized cells (REIC) gene was cloned, and an analysis revealed that it is identical to the Dickkopf-3 (Dkk-3) gene, which is a member of the Dickkopf gene family (1). REIC/Dkk-3 expression is down-regulated in a broad range of cancer cell lines and clinical cancer materials (2-10). The expression of the human REIC/Dkk-3 gene is significantly down-regulated in human prostate cancer specimens in comparison to normal human prostate tissue at the protein level (5). In addition, REIC/Dkk-3 expression in prostate cancer is consistently down-regulated at the critical transition from non-invasive disease to highly invasive disease $(5,6)$.

Forced expression of REIC/Dkk-3, using a plasmid vector, inhibits cell growth in HeLa, liver cancer and glioma cell lines $(4,11)$. Adenovirus vector mediated overexpression of REIC/ Dkk-3 selectively induces apoptosis in prostatic cancer cells through the activation of c-Jun- $\mathrm{NH}_{2}$-kinase (JNK) and c-Jun but not in non-cancer cells (5). Adenovirus mediated REIC/ Dkk-3 overexpression inhibits the expression of Id-1, which works in cell cycle progression, anti-apoptosis and angiogenesis (12) in malignant mesothelioma cells (9). These findings strongly suggest that REIC/Dkk-3 plays a role in the inhibition of tumor progression in a broad range of human malignancies. Although the ectopic REIC/Dkk-3 overexpression by adenovirus and plasmid vector induces cell death in cancer cells, the ability of continuously expressed REIC/Dkk-3 to affect the tumorigenic processes and to work as a tumor suppressor remains to be elucidated.

This study explored the anti-cancer effects of continuous REIC/Dkk-3 expression using stable transfectant prostate cancer cells. In addition, a proteomics analysis of the REIC/ Dkk-3 and control clones was performed to clarify the anticancer molecular mechanism of the REIC/Dkk-3 stable expression. 


\section{Materials and methods}

Cell culture. The RM9 mouse prostate cancer cell line was kindly provided by Professor T.C. Thompson (The University of Texas, M.D. Anderson Cancer Center, Houston, TX). The cell line was derived from a primary prostate tumor induced in the Zipras/myc-9 infected mouse prostate reconstitution (MPR) model system of C57BL/6 mice (13). The cells were grown in Dulbecco's modified Eagle's medium (DMEM; Invitrogen, Carlsbad, CA) supplemented with $10 \%$ fetal bovine serum (FBS; Biowest, Nuaille, France), penicillin (50 IU/ml) and streptomycin $(50 \mu \mathrm{g} / \mathrm{ml})$ under a humidified atmosphere of $5 \% \mathrm{CO}_{2}$ at $37^{\circ} \mathrm{C}$ and split at a $1: 5$ ratio every three days.

Stable transfection of RM9 cells with REIC/Dkk-3. A panel of RM9 cell clones expressing REIC/Dkk-3 (RM9-REIC) was established by stable transfection with a sense expression plasmid. A plasmid of pcDNA3 (Invitrogen) containing the human REIC/Dkk-3 gene and the selectable marker gene for neomycin resistance, was used for REIC/Dkk-3 clones. As the control, RM9 cells received pcDNA3 alone and then empty vector-transfected control clone (RM9-EV) was also established. Transfections were performed using Lipofectamine reagent (Invitrogen) according to the manufacturer's protocol. Cells were treated with each plasmid for $6 \mathrm{~h}$ and the medium was exchanged to the flesh complete medium. At $48 \mathrm{~h}$ after transfection, cells were placed into selection medium containing $500 \mu \mathrm{g} / \mathrm{ml} \mathrm{G} 418$ (Sigma, St. Louis, MO). After 21 days of selection, individual G418-resistant colonies were subcloned and the stable transfectants were stocked in liquid nitrogen.

Western blot analysis. The cells were lysed in ice-cold lysis buffer [20 mM Tris ( $\mathrm{pH} 7.5$ ), $150 \mathrm{mM} \mathrm{NaCl}, 1 \mathrm{mM} \mathrm{Na} 2$ EDTA, $1 \mathrm{mM}$ EGTA, $1 \%$ Triton, $2.5 \mathrm{mM}$ sodium pyrophosphate, $1 \mathrm{mM}$ ß-glycerophosphate, $1 \mathrm{mM} \mathrm{Na} \mathrm{VO}_{4}, 1 \mu \mathrm{g} / \mathrm{ml}$ leupeptin]. Insoluble fragments were removed by centrifugation at $16,000 \mathrm{x} \mathrm{g}$ for $10 \mathrm{~min}$ at $4^{\circ} \mathrm{C}$ and the supernatant lysate was then immediately shock frozen and stored at $-80^{\circ} \mathrm{C}$. The protein concentrations were determined using a protein assay kit (Bio-Rad, Hercules, CA).

Extracted cell protein $(\sim 50 \mu \mathrm{g})$ was mixed with $6 \mathrm{X}$ loading buffer [450 mM Tris (pH 6.8), 45\% sucrose, 10\% ß-mercaptoethanol, 15\% SDS, and bromophenol blue] and separated in SDS-PAGE gradient gels (Bio-Rad). The proteins were transferred onto PVDF membranes in buffer with $25 \mathrm{mM}$ Tris, $192 \mathrm{mM}$ glycine and $20 \%$ ethanol. Following the transfer, the membranes were blocked with $5 \%$ nonfat dry milk in phosphate buffered saline $0.1 \%$ Tween-20 and probed with the antibodies specified as follows. A rabbit polyclonal anti-REIC/Dkk-3 antibody was previously made in this laboratory (5). Horseradish peroxidase-conjugated secondary antibodies and ECL substrate kit (GE Healthcare, Buckinghamshire, UK) were used to detect the protein.

Apoptosis assay and cell proliferation assay. The apoptotic cells were determined by Hoechst 33342 staining under fluorescence microscopy and the apoptotic cell rate was calculated (10). The dye solution was added into the culture medium and then cells were incubated for $10 \mathrm{~min}$. Cells were directly observed with phase contrast and fluorescence microscopy. The apoptotic cells were identified by the presence of highly condensed or fragmented nuclei. Apoptotic cells were counted in three different fields using microscopic observations. One hundred cells were judged under one field.

To analyze cell proliferation in each cell clone, the viability of RM9 cells was determined at the indicated time points. Briefly, cells were seeded at the concentration of $10^{5}$ cells and cultured in a flat-bottom 6-well dish. Using CellTiter $96^{\circledR}$ Aqueous One Solution Cell Proliferation Assay (Promega Corp., Madison, WI), the cell viability was then estimated by measuring the absorbance at $490 \mathrm{~nm}$ according to the manufacturer's instructions.

In vivo-tumorigenesis assay of REIC/Dkk-3 stable transfectants. Adult male C57BL/6 mice purchased from Charles River Laboratories (Yokohama, Japan) were used for the tumorigenesis experiments. Animal care, human use and treatment of mice were in strict compliance with the guidelines of Okayama University. The parental cells and clones $\left[5 \times 10^{3}\right.$ cells in $10 \mu 1$ of phosphate-buffered saline (PBS)] were orthotopically injected into the right lobe of the dorsal prostate. On day 14 after cell injection, mice were sacrificed and the weight of the dissected tumor was measured.

Two-dimensional polyacrylamide gel electrophoresis (2D-PAGE). RM9-EV and RM9-REIC1 cells were suspended in ice-cold sample buffer containing $40 \mathrm{mM}$ Tris, $7 \mathrm{M}$ urea, $2 \mathrm{M}$ thiourea, 4\% CHAPS, $100 \mathrm{mM}$ 1,4-dithioerythritol, and protease inhibitor cocktail (Roche, Mannheim, Germany). The suspensions were incubated for $60 \mathrm{~min}$ on ice and centrifuged at $13,000 \mathrm{x}$ for $10 \mathrm{~min}$. The supernatant of each clone was used for each electrophoresis. Total protein $(20 \mu \mathrm{g})$ in sample buffer was applied to immobilized $\mathrm{pH}$ gradient ( $\mathrm{pH} 3-10)$ nonlinear strips. Isoelectrofocusing was carried out by increasing the voltage in a stepwise manner up to $12,000 \mathrm{~V}$, and then continuing for $2 \mathrm{~h}$ at $12,000 \mathrm{~V}$. The second dimension was carried out in a 9-16\% linear gradient polyacrylamide gel at $40 \mathrm{~mA}$ at a constant current for $\sim 5 \mathrm{~h}$, until the dye front reached the bottom of the gel.

Protein visualization and image analysis. After protein fixation in $40 \%$ methanol and $5 \%$ phosphoric acid for $12 \mathrm{~h}$, the gels were stained using a Dodeca Silver Stain Kit (Bio-Rad) according to the manufacturer's instructions. The gels were destained using the Silver Stain MS Kit (Wako, Osaka, Japan), and then were scanned in a Gel Doc XR 170-8170 (Bio-Rad). All data were converted into electronic files, which were then analyzed using the Finger printing II Software program (Bio-Rad).

Protein spot identification. For mass spectrometry fingerprinting, protein spots were directly cut out of gels, destained, and treated with trypsin. The aliquots of the peptide mixtures obtained by trypsin treatment were applied to a target disk and then allowed to air-dry. Spectra were obtained by liquid chromatography-tandem mass spectrometry using the LC/MSD Trap XCT Ultra (Agilent Technologies, Santa Clara, CA, USA). The protein database search was performed using the Spectrum Mill Ms Proteomics Workbench software program (http://www.proteomesoftware.com). 
A

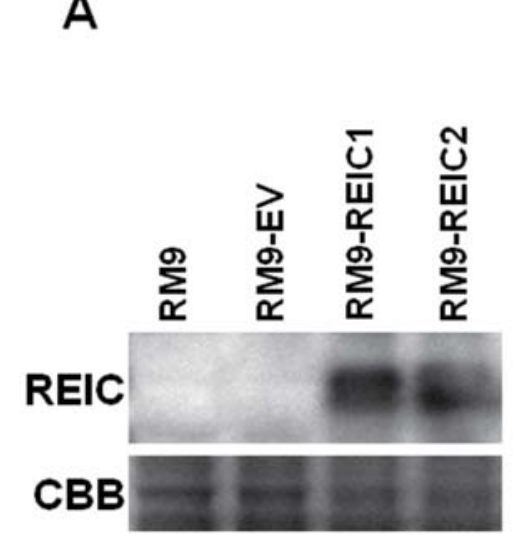

B


Hoechist staining

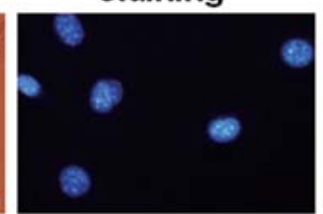

Higher

magnification
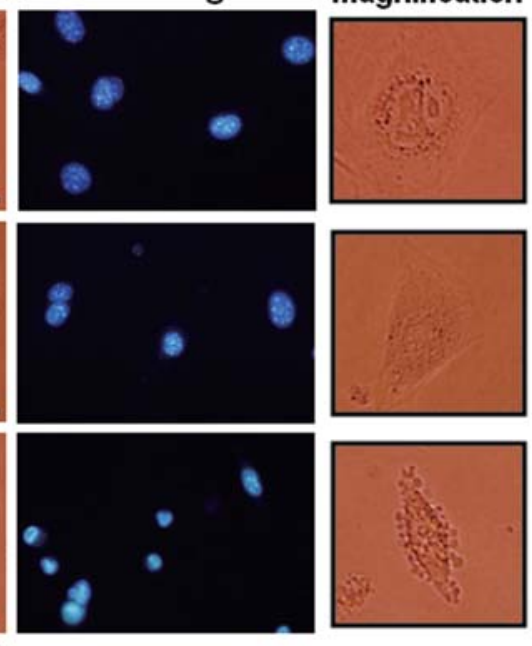

Figure 1. (A) REIC/Dkk-3 expression was analyzed by Western blot analysis in the parental, RM9-EV and RM9-REIC clones. (B) The clone cells were observed under phase contrast and fluorescence microscopy in standard culture conditions. The cell nucleus was stained with Hoechst 33342 . The image of higher magnification shows a selected area (black square) in the phase contrast panel.

Statistical analysis. The data are presented as the mean $\pm \mathrm{SE}$ Unpaired Student's t-test was performed for statistical analysis between the two groups and a difference of $\mathrm{p}<0.05$ was considered significant.

\section{Results}

REIC/Dkk-3 stable transfectants show an anti-cancer phenotype in RM9 cancer cells. In order to analyze the effects of continuously expressed REIC/Dkk-3 in cancer cells, the expression of REIC/Dkk-3 was stably restored in the mouse prostate cancer RM9 cells. Successful re-expression of REIC/Dkk-3 in the RM9-REIC cell clones was confirmed by Western blot analysis (Fig. 1A). Since the used REIC/Dkk-3 antibody recognizes both human and mouse REIC/Dkk-3 protein (8), no mouse REIC/Dkk-3 expression was seen in the parental and control RM9-EV cells.

Microscopic observations indicate the most striking features of the RM9-REIC clones to be cell shrinking and membrane blebbing (Fig. 1B), which are the characteristics of cellular stress (14). To evaluate the incidence of apoptotic cell death in the REIC/Dkk-3 stable transfectants, the nuclei were visualized by Hoechst 33342 staining under standard culture condition. The nuclei of RM9-REIC cells appeared to be smaller in comparison to that of the controls (Fig. 1B). The incidence of apoptosis presented with highly condensed or fragmented nuclei was significantly increased in the RM9-REIC clones in comparison to the controls (Fig. 2). These observations indicate that stable REIC/Dkk-3 expression in RM9 cells induces an anti-cancer phenotype in terms of the incidence of cell death.

The cell proliferation of each clone was analyzed by measuring cell viability. The proliferation of RM9-REIC clones was significantly decreased in comparison to the RM9-EV and parental cells (Fig. 3), thus indicating the reduced cell growth in the REIC/Dkk-3 stable transfectants.

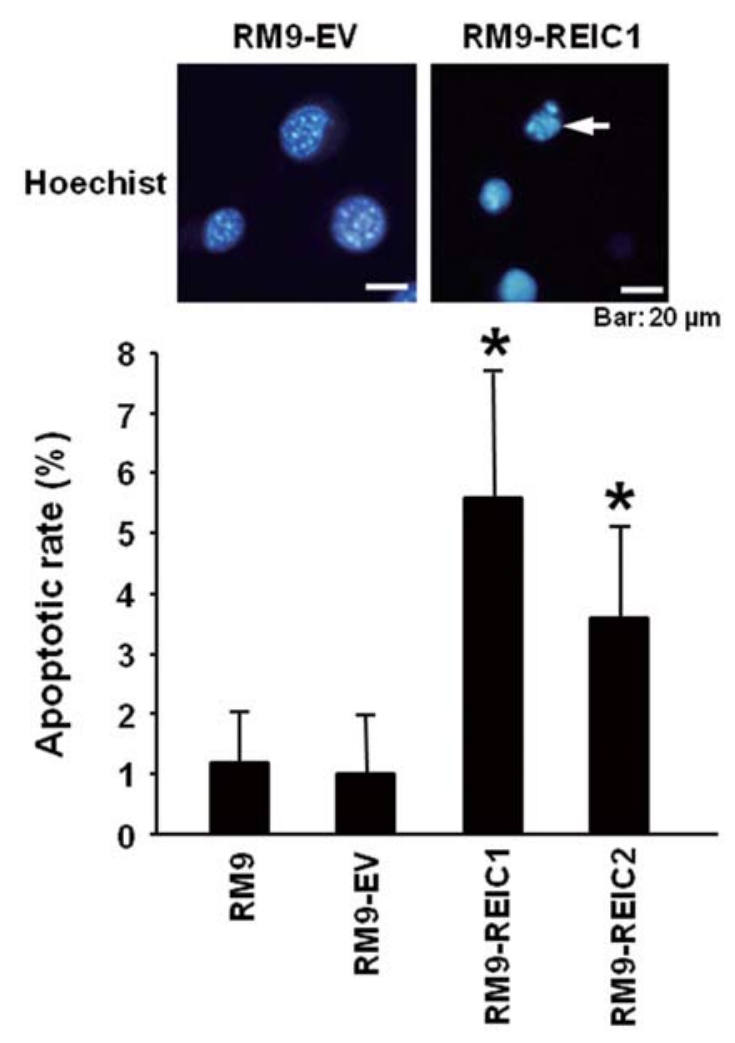

Figure 2. The effect of REIC/Dkk-3 stable expression on the apoptotic incidence was examined in vitro. Cell nucleus was stained with Hoechst 33342 and representative fluorescent image is shown in the upper panel. White arrow indicates fragmented nucleus. The percentage of apoptotic cells was determined for each clone. *Significant difference observed between RM9-REIC and RM9-EV clones. The number of observations was five in each clone.

To examine the effect of continuous REIC/Dkk-3 expression in in vivo tumor growth, the RM9-REIC cells were orthotopically injected in the mouse model. The tumor growth of 




Figure 3. The effect of REIC/Dkk-3 stable expression on the cell proliferation was examined in vitro. The cell viability assay was done in each clone by measuring the absorbance at $490 \mathrm{~nm}$. "Significant difference observed between RM9-REIC and RM9-EV clones. The number of observations was eight in each clone.
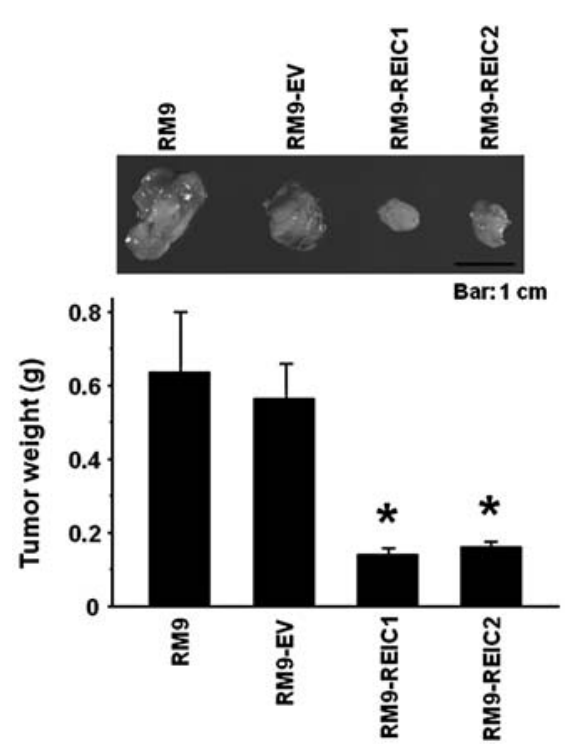

Figure 4. The effect of REIC/Dkk-3 stable expression on the RM9 tumor growth was examined using an in vivo mouse model. The orthotopic tumors were dissected on day 14 after cell injection. A representative macroscopic view of the tumors is shown in the upper panel. The weight of dissected tumors was compared between the groups. Each group of the cell clone consisted of 4 or 5 mice. "Significant difference observed between RM9-REIC and RM9-EV derived tumors.

REIC/Dkk-3 stably transfected RM9 cells was significantly inhibited in comparison to those of the control cells (Fig. 4).

Differentially expressed proteins between RM9-REIC and the control RM9-EV cells. Two-dimensional electrophoresis was used to identify the differentially expressed proteins between RM9-REIC and RM9-EV cells. The proteins were extracted directly from cultured cells with the lysis buffer and separated by 2D-PAGE. Protein spots ( 300) were visualized on each gel by silver staining (Fig. 5). Comparison of these proteins on 2D gels revealed that nine protein spots were reproducibly changed. A spot volume $>1.5$-fold of that for RM9-EV cells was considered a meaningful increase, and a spot volume of $<50 \%$ was considered a meaningful decrease. Those proteins were identified by peptide mass fingerprinting using mass

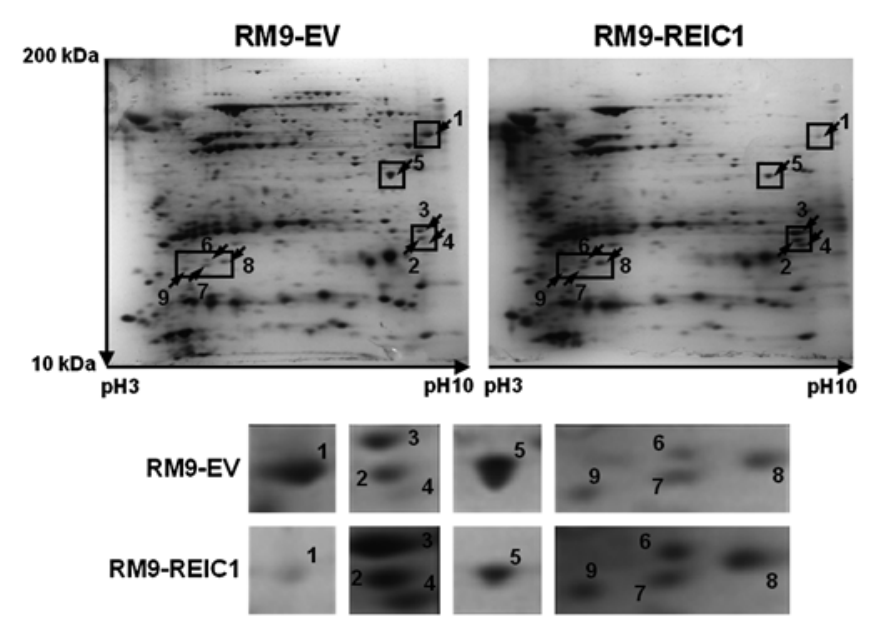

Figure 5. Comparative proteomic analysis between RM9-REIC and RM9-EV cells. Proteins were extracted and each protein sample was resolved by 2D-PAGE. The gels were stained with a silver staining kit. Differentially expressed proteins are numbered from 1 to 9 and the spots are indicated with arrows. Lower panel, spots enlarged in boxes and compared between RM9-REIC and RM9-EV cells.

spectrometry and a database search (Table I). The seven increased proteins in RM9-REIC were peroxiredoxin-1, GST-P1 (glutathione S-transferase P1), transgelin-2, MRP-L12 (39S ribosomal protein L12), aci-reductone dioxygenase (ARD), GRP78 (78-kDa glucose-regulated protein) and Sorcin, whereas the two decreased proteins were eEF1A-1 (eukaryotic elongation factor 1A-1) and cyclophilin-40 in comparison to the RM9-EV cells.

\section{Discussion}

REIC/Dkk-3 is a secretory protein and it is significantly downregulated in cancer cell lines and more pathologically malignant tumors (1-10). The expression is barely detected in human prostate cancer cell lines, but is abundant in the primary epithelial cells $(5,6)$. The current study demonstrates that the continuous overexpression of REIC/Dkk-3 induces a cell phenotype characterized by a higher apoptotic incidence and anti-proliferative status in the RM9 prostate cancer cells. The suppression of tumor growth was further confirmed in an in vivo situation using an orthotopic tumor model. These phenomena were consistent with other published studies (3,5,9,10,15-18), showing that ectopic expression of REIC/ Dkk-3 induces apoptosis and inhibits cell proliferation in various cancer cells. Therefore, the lack or absence of endogenous REIC/Dkk-3 expression in RM9 cancer cells seems to be important for the cellular effects induced by the REIC/Dkk-3 overexpression.

In order to discover the differently expressed proteins among the RM9-REIC and control clones, total proteins were separated by 2D-PAGE and were identified by peptide mass fingerprinting. Nine proteins were differentially expressed between the clones, and these can be largely categorized into classes such as protein synthesis, detoxification, metabolism, and cytoskeleton based on their functions. Among them, GRP78 (also called BiP) is revealed to be up-regulated. GRP78 protein is associated with protein folding at the endoplasmic reticulum (ER) and is a key signaling molecule of 
Table I. Identification of the proteins differentially expressed between RM9-REIC and RM9-EV clonal cells.

\begin{tabular}{llcccc}
\hline Spot No. & Protein name & Accession code & Differential ratio & MW (kDa)/pI & Function \\
\hline 1 & eEF1A-1 & P10126 & -21.9 & $50.1 / 9.10$ & Protein synthesis \\
2 & peroxiredoxin-1 & P35700 & +11.6 & $22.2 / 8.26$ & Detoxification \\
3 & GST-P1 & P19157 & +21.6 & $23.6 / 7.69$ & Detoxification \\
4 & transgelin-2 & Q9WVA4 & +25.4 & $23.6 / 6.59$ & Cytoskeleton \\
5 & cyclophilin-40 & Q9CR16 & -6.1 & $40.7 / 7.07$ & Protein folding \\
6 & MRP-L12 & Q9DB15 & +5.7 & $21.7 / 9.34$ & Protein synthesis \\
7 & ARD & Q99JT9 & +1.6 & $21.5 / 5.31$ & Metabolism \\
8 & GRP78 & P20029 & +2.4 & $72.4 / 5.07$ & Protein folding \\
9 & Sorcin & Q6P069 & +1.9 & $21.6 / 5.32$ & Ca \\
\hline
\end{tabular}

eEF1A-1, Eukaryotic elongation factor 1A-1; GST-P1, glutathione S-transferase P1; MRP-L12, 39S ribosomal protein L12; ARD, aci-reductone dioxygenase; GRP78, 78-kDa glucose-regulated protein. The accession codes refer to the Entrez Nucleotide Database of the National Center for Biotechnology Information. Differential ratio indicates the fold change of the expression level in RM9-REIC cells in comparison to RM9-EV cells.

ER stress $(19,20)$. ER stress can be evoked and plays a role in the induction of apoptosis when particular glycosylated proteins are overexpressed and the protein folding and secretion are impaired $(21,22)$. Interestingly, previous studies disclosed that REIC/Dkk-3 overexpression by an adenovirus vector triggered the up-regulation of GRP78 and ER stress triggered apoptosis in the REIC absent mesothelioma $211 \mathrm{H}$ cancer cells (9) and prostate cancer PC3 cells (unpublished data). In addition, the apoptosis induction by REIC/Dkk-3 could be regulated through the molecular mechanism of JNK phosphorylation accompanied with ER stress $(5,9,16)$. Therefore, ER stress definitely participated in the mechanism of REIC/Dkk-3 induced apoptosis in the cancer cells. It is probable that the phenotype observed in the RM9-REIC clones is induced partially by the ER stress-mediated signaling.

Stable REIC/Dkk-3 expression induced an anti-cancer phenotype in vivo. There is accumulating evidence that the intracellular expression of REIC/Dkk-3 is negatively related to the development of a malignant and/or atypical cell phenotype $(3,4,6,15,23,24)$. REIC/Dkk-3 overexpression in cancer cells inhibits the invasive and motile cell phenotype in vitro $(23,24)$ and metastasis in vivo $(15)$. siRNA-mediated depletion of REIC/Dkk-3 disrupts in vitro acinar morphogenesis and cell polarization in RWPE-1 cells which originate from human normal prostate epithelium (6). These findings suggest that the expression of REIC/Dkk-3 in normal cells prevents the development of cancer cells and decreased REIC/Dkk-3 expression is involved in the tumorigenesis process. Interestingly, the current proteomics analysis disclosed that the expression of eEF1A-1, which could work as an oncogene (25), is significantly suppressed in the RM9-REIC clone. From this point of view, it is conceivable that stable REIC/Dkk-3 expression may down-regulate the signaling of eEF1A-1 and then suppress the tumorigenesis of RM9 prostate cancer cells.

Although REIC/Dkk-3 is a tumor suppressor in a broad range of cancers, the molecular mechanism of anti-tumorigenesis has not been elucidated. The current study shows that continuously expressed REIC/Dkk-3 works as a tumor suppressor, as well as in the case of ectopic overexpression. Therefore, cells with less intracellular REIC/Dkk-3 might have more advantages of growth. If so, the REIC/Dkk-3 down-regulated cells would be selected to survive and grow as clonal cells, thereby occupying a large part of the cancer lesions. This theory may account for the reason why so many types of cancer cells show a lack of REIC/Dkk-3 expression. An important future step is to clarify the molecular function of REIC/Dkk-3 and the current results of proteomic screening may be useful for the analysis of the signaling pathway.

\section{Acknowledgements}

This work was supported by a grant from the Ministry of Education, Culture, Sports, Science and Technology's FY2006 'Creation of Innovation Centers for Advanced Interdisciplinary Research Areas' Scheme in Japan. We also thank Hideo Ueki (Okayama University) for his valuable technical assistance.

\section{References}

1. Tsuji T, Miyazaki M, Sakaguchi M, Inoue Y and Namba M: A REIC gene shows down-regulation in human immortalized cells and human tumor-derived cell lines. Biochem Biophys Res Commun 268: 20-24, 2000.

2. Nozaki I, Tsuji T, Iijima O, Ohmura Y, Andou A, Miyazaki M, Shimizu N and Namba M: Reduced expression of REIC/Dkk-3 gene in non-small cell lung cancer. Int J Oncol 19: 117-121, 2001.

3. Tsuji T, Nozaki I, Miyazaki M, Sakaguchi M, Pu H, Hamazaki Y, Iijima O and Namba M: Antiproliferative activity of REIC/Dkk-3 and its significant down-regulation in non-small-cell lung carcinomas. Biochem Biophys Res Commun 289: 257-263, 2001.

4. Hsieh SY, Hsieh PS, Chiu CT and Chen WY: Dickkopf-3/REIC functions as a suppressor gene of tumor growth. Oncogene 23: 9183-9189, 2004.

5. Abarzua F, Sakaguchi M, Takaishi M, Nasu Y, Kurose K, Ebara S, Miyazaki M, Namba M, Kumon H and Huh NH: Adenovirusmediated overexpression of REIC/Dkk-3 selectively induces apoptosis in human prostate cancer cells through activation of c-Jun-NH2-kinase. Cancer Res 65: 9617-9622, 2005.

6. Kawano Y, Kitaoka M, Hamada Y, Walker MM, Waxman J and Kypta RM: Regulation of prostate cell growth and morphogenesis by Dickkopf-3. Oncogene 25: 6528-6537, 2006. 
7. Tanimoto R, Abarzua F, Sakaguchi M, Takaishi M, Nasu Y, Kumon $\mathrm{H}$ and Huh NH: REIC/Dkk-3 as a potential gene therapeutic agent against human testicular cancer. Int $\mathrm{J}$ Mol Med 19: 363-368, 2007.

8. Abarzua F, Sakaguchi M, Tanimoto R, Sonegawa H, Li DW, Edamura K, Kobayashi T, Watanabe M, Kashiwakura Y, Kaku H, Saika T, Nakamura K, Nasu Y, Kumon H and Huh NH: Heat shock proteins play a crucial role in tumor-specific apoptosis by REIC/Dkk-3. Int J Mol Med 20: 37-43, 2007.

9. Kashiwakura Y, Ochiai K, Watanabe M, Abarzua F, Sakaguchi M, Takaoka M, Tanimoto R, Nasu Y, Huh NH and Kumon H: Down-regulation of inhibition of differentiation-1 via activation of activating transcription factor 3 and Smad regulates REIC/ Dickkopf-3-induced apoptosis. Cancer Res 68: 8333-8341, 2008.

10. Kawasaki K, Watanabe M, Sakaguchi M, Ogasawara Y, Ochiai K, Nasu Y, Doihara H, Kashiwakura Y, Huh NH, Kumon H and Date H: REIC/Dkk-3 overexpression downregulates P-glycoprotein in multidrug-resistant MCF7/ADR cells and induces apoptosis in breast cancer. Cancer Gene Ther 16: 65-72, 2009.

11. Mizobuchi Y, Matsuzaki K, Kuwayama K, Kitazato K, Mure H, Kageji T and Nagahiro S: REIC/Dkk-3 induces cell death in human malignant glioma. Neuro Oncol 10: 244-253, 2008.

12. Ling MT, Wang X, Zhang X and Wong YC: The multiple roles of Id-1 in cancer progression. Differentiation 74: 481-487, 2006.

13. Thompson TC, Southgate J, Kitchener G and Land H: Multistage carcinogenesis induced by ras and myc oncogenes in a reconstituted organ. Cell 56: 917-930, 1989.

14. Leverrier Y and Ridley AJ: Apoptosis: caspases orchestrate the ROCK 'n' bleb. Nat Cell Biol 3: E91-E93, 2001.

15. Edamura K, Nasu Y, Takaishi M, Kobayashi T, Abarzua F, Sakaguchi M, Kashiwakura Y, Ebara S, Saika T, Watanabe M, Huh NH and Kumon H: Adenovirus-mediated REIC/Dkk-3 gene transfer inhibits tumor growth and metastasis in an orthotopic prostate cancer model. Cancer Gene Ther 14: 765-772, 2007.

16. Abarzua F, Kashiwakura Y, Takaoka M, Watanabe M, Ochiai K Sakaguchi M, Iwawaki T, Tanimoto R, Nasu Y, Huh NH and Kumon H: An N-terminal 78 amino acid truncation of REIC/Dkk-3 effectively induces apoptosis. Biochem Biophys Res Commun 375: 614-618, 2008.
17. Sakaguchi M, Kataoka K, Abarzua F, Tanimoto R, Watanabe M, Murata H, Than SS, Kurose K, Kashiwakura Y, Ochiai K, Nasu Y, Kumon $\mathrm{H}$ and Huh NH: Overexpression of REIC/Dkk-3 in normal fibroblasts suppresses tumor growth via induction of IL-7. J Biol Chem 284: 14236-14244, 2009.

18. Watanabe M, Kashiwakura Y, Huang P, Ochiai K, Futami J, Li SA, Takaoka M, Nasu Y, Sakaguchi M, Huh NH and Kumon H: Immunological aspects of REIC/Dkk-3 in monocyte differentiation and tumor regression. Int J Oncol 34: 657-663, 2009.

19. Shen J, Chen X, Hendershot L and Prywes R: ER stress regulation of ATF6 localization by dissociation of BiP/GRP78 binding and unmasking of Golgi localization signals. Dev Cell 3: 99-111, 2002

20. Christis C, Lubsen NH and Braakman I: Protein folding includes oligomerization - examples from the endoplasmic reticulum and cytosol. FEBS J 275: 4700-4727, 2008.

21. Herr I and Debatin KM: Cellular stress response and apoptosis in cancer therapy. Blood 98: 2603-2614, 2001.

22. Cudna RE and Dickson AJ: Endoplasmic reticulum signaling as a determinant of recombinant protein expression. Biotechnol Bioeng 81: 56-65, 2003.

23. Hoang BH, Kubo T, Healey JH, Yang R, Nathan SS, Kolb EA, Mazza B, Meyers PA and Gorlick R: Dickkopf 3 inhibits invasion and motility of Saos-2 osteosarcoma cells by modulating the Wnt-beta-catenin pathway. Cancer Res 64: 2734-2739, 2004

24. Saeb-Parsy K, Veerakumarasivam A, Wallard MJ, Thorne N, Kawano Y, Murphy G, Neal DE, Mills IG and Kelly JD: MT1MMP regulates urothelial cell invasion via transcriptional regulation of Dickkopf-3. Br J Cancer 99: 663-669, 2008.

25 Lamberti A, Caraglia M, Longo O, Marra M, Abbruzzese A and Arcari P: The translation elongation factor $1 \mathrm{~A}$ in tumorigenesis, signal transduction and apoptosis: review article. Amino Acids 26: 443-448, 2004 